\title{
LA METACOGNICIÓN COMO ESTRATEGIA PARA DETECTAR PROCESOS COGNITIVOS EN NIÑOS CON RETRASO MENTAL
}

\author{
Ana Patricia Vázquez Chaves ${ }^{1}$ \\ Suzanne León Rodríguez ${ }^{2}$ \\ María Judith Rodríguez Ramírez ${ }^{3}$
}

\section{Resumen}

Este artículo se basa en una investigación tendiente a detectar los procesos cognitivos que empleaban los niños ${ }^{4}$ con retraso mental en la resolución de situaciones matemáticas, mediante el uso de estrategias metacognitivas. Se trabajó con una muestra de veinte niños con retraso mental que asisten a aulas integradas, de las provincias de Heredia y Alajuela. Se elaboró la Batería de Resolución de Situaciones Matemáticas para Niños con Retardo Mental (BARSIMAR) para detectar los procesos cognitivos que empleaban los niños ante la ejecución de las estrategias metacognitivas. Los resultados demues- tran que entre mayor sea el compromiso cognitivo de los niños mayor dificultad presentan al emplear los procesos cognitivos y al registrar la información en la memoria a corto plazo. Los niños más avanzados lograron emplear mejor los procesos, pero con interferencias y dificultades para recuperar la información de la memoria a largo plazo y dar respuestas adecuadas ante la tarea cognitiva que tiene en frente.

\section{Abstract}

It was planned to detect the cognitive processes that mentally retarded children used when solving math problems,

\footnotetext{
${ }^{1}$ Educadora especial, Retraso Mental y Dificultades del Aprendizaje. Universidad de Costa Rica, Universidad Nacional y Pontificia Universidad Católica de Chile. Cargos: docente en la UNA impartiendo cursos en bachillerato y licenciatura de la carrera de Educación Especial y directora de trabajos finales de graduación. Desarrollo de proyectos de investigación. Docente de Aula Integrada Escuela Jesús Jiménez Zamora, Tibás.

2 Licenciada en Educación Especial con énfasis en Integración, Universidad Nacional. Cargos: docente de Apoyo Itinerante en Parálisis Cerebral y docente en Aula Integrada. Lugar de trabajo: Alajuela, en la Escuela Líder Pacto del Jocote.

${ }^{3}$ Licenciatura en Educación Especial con énfasis en Integración, Universidad Nacional. Cargos: Maestra de Educación Especial.

${ }^{4}$ Solamente para propósito de sencillez en el estilo, este documento utiliza un formato radicional que no contempla las diferencias de género. La posición es clara y firme respecto a que toda discriminación sobre esta base o de cualquier otra naturaleza se considera contraria a los principios que aquí se expresan.
} 
through the use or metacognitive strategies. The work was done whit a sample of twenty mentally retarded children that attend integrated classrooms from of provinces of Heredia and Alajuela. An instrument called Batería de Resolución de Situaciones Matemáticas para Niños con Retardo Mental (BARSIMAR) was used to detect the cognitive processes that children used when working with metacognitive strategies. The results show that the more cognitive engaged the children are, the more difficulty they will have when using cognitive processes to register the informa- tion in the short term memory. The advanced kids could use the processes in a better way, but with interferences and difficulty to recover the information from the long term memory and give correct answers to the cognitive task they are working on.

\section{Palabras claves}

Metacognición, estrategias metacognitivas, estructuras y procesos cognitivos, procesamiento de la información, Resolución de Situaciones Matemáticas, retraso mental.

\section{Introducción}

$\mathrm{L}$

a metacognición es hacer pensamiento sobre el propio pensamiento y una forma de ayudar a los niños a tomar conciencia de sus propios procesos cognitivos para la aplicación en su vida cotidiana. El presente artículo se basa en una investigación diseñada desde la teoría cognitiva y el enfoque del procesamiento de la información. Estudios realizados en otros países, en I y II ciclos de Educación General Básica, en secundaria y con adultos (Escudero, 2002; Bañuelos, 2002; González, s.f., García, Montero, Romero y Salvador, 1996), reflejan su importancia como estrategia de mediación. En Costa Rica, no se reportan estudios que precedan el uso de estrategias metacognitivas en el área de educación especial, de ahí su trascendencia.

Dockrell y McShane (1997) plantean que en los niños con dificultades de aprendizaje y desde una perspectiva cognitiva, las estrategias para almacenar, adquirir o utilizar el conocimiento se desarrollan a un ritmo más lento. Conocer los procesos cognitivos que utilizan estos niños al resolver situaciones matemáticas, sirve para planificar mejores y más adecuadas estrategias metodológicas y estimular la metacognición en todas las áreas de su aprendizaje.

En problema que se planteó fue el siguiente: ¿Cuáles son los procesos cognitivos que emplean los niños con retraso mental, que asisten a aulas integradas, en la resolución de situaciones matemáticas utilizando las estrategias metacognitivas?

En el transcurso de la investigación, emergió una serie de preguntas directrices, propias de los estudios cualitativos, que permitieron profundizar y orientar el tema de estudio. Se pretendió analizar los procesos cognitivos que 
empleaban los niños con retraso mental que asisten a aulas integradas, en la Resolución de Situaciones Matemáticas mediante el uso de estrategias metacognitivas. Los objetivos específicos estaban orientados a identificar los conocimientos previos que tienen los niños con retraso mental, respecto a la Resolución de Situaciones Matemáticas, mediante la aplicación de estrategias metacognitivas y su respectivo análisis. También se buscó construir una Batería de Estrategias Metacognitivas, que estimulara la expresión de los procesos cognitivos que usan los niños con retraso mental en la Resolución de Situaciones Matemáticas, según su nivel de funcionamiento y los conocimientos previos identificados. Posteriormente se propuso identificar los procesos cognitivos que emplean los niños con retraso mental en la Resolución de Situaciones Matemáticas, aplicando individualmente la Batería de Resolución de Situaciones Matemáticas para Niños con Retardo Mental (BARSIMAR). Por último, se determinaron los procesos cognitivos que utilizaban los niños con retraso mental, al emplear estrategias metacognitivas en la Resolución de Situaciones Matemáticas.

La Psicología Cognitiva se basa en los procesos mentales, ya que se concentra en la forma en la que los niños descubren, interpretan, almacenan y recuperan la información. Dependiendo de la estimulación que reciben los niños y las posibilidades de relacionar lo nuevo con lo que ya conocen, van a demostrar diferentes formas de desarrollar su aprendizaje.

Se debe buscar la forma de reforzar el conocimiento construyendo personas pensantes, que demuestren y expresen todo su aprendizaje y conocimiento adquirido, se deben considerar sus motivaciones, para que avancen y exploten todas sus habilidades según su ritmo y capacidad; así van adquiriendo conciencia de su propio aprendizaje y logran autorregular cada una de las tareas cognitivas que se les presentan.

En el enfoque del procesamiento de la información, existen dos orientaciones distintas: la de las estructuras cognitivas y la de los procesos cognitivos. En la primera, se presentan tres grandes estructuras o almacenes de memoria que son: registro sensorial, memoria a corto plazo o de trabajo y la memoria a largo plazo. El grupo de investigación se centró en los procesos dinámicos implicados en el uso de la memoria para buscar y recuperar adecuadamente la información y no en las estructuras cognitivas.

Los procesos involucrados en el procesamiento de la información, y que constituyen el eje central del estudio, son: la recepción de los estímulos recibidos, la percepción selectiva de los estímulos, el almacenamiento en la memoria a corto plazo, la codificación semántica, el almacenamiento en la memoria a largo plazo, la búsqueda y recuperación de la información, el organizador de respuesta y la retroalimentación y refuerzo.

Cada uno de estos procesos cognitivos es responsable de procesar, almacenar y utilizar la información proveniente del entomo, según las demandas 
que la tarea cognitiva le exige al estudiante o persona que la ejecuta. Los procesos iniciales reciben y seleccionan los estímulos que se relacionan con la tarea cognitiva; los procesos siguientes se encargan de comparar, integrar y practicar la información en la memoria a corto plazo y, finalmente, los últimos procesos son los responsables de buscar, recuperar, aplicar y evaluar la información contenida en la memoria a largo plazo.

Es importante destacar que el grupo de investigación construyó un esquema donde se puede observar detalladamente el flujo de la información. En él se puede ver la organización de las estructuras y los procesos que la componen, las relaciones entre ellos, así como el recorrido que hace la información desde la entrada hasta la salida del sistema. El modelo de procesamiento de información presentado permite entender el funcionamiento normal de una persona y al mismo tiempo comprender como los niños con discapacidad perciben la información, de forma más complicada de lo que se piensa, se les dificulta recordar lo aprendido y almacenar la información en la memoria y, por lo tanto, es difícil que se logre un aprendizaje eficaz.

La metacognición se entiende como una estrategia cognitiva que implica la autoconciencia del proceso de aprendizaje. El aprendizaje es un proceso metacognitivo o autorregulado. Es autorregulado porque se busca un aprendizaje eficaz, donde el estudiante pueda monitorear su propio proceso, para que así pueda adoptar decisiones apropiadas y mantenerse motivado y concentrado en la tarea.

La Resolución de Situaciones Matemáticas es una concepción menos tradicional que la Resolución de Problemas Matemáticos; desde una perspectiva más actualizada y para efectos de este estudio, se adopta el primer nombre. La diferencia terminológica es importante, ya que la palabra 'problema' conlleva una connotación negativa que, eventualmente, podría significar para el niño algún tipo de bloqueo a la hora de resolver la tarea cognitiva que tiene al frente. El término "situación matemática" es más amplio y puede ser aplicado a otras situaciones tanto académicas como de la vida cotidiana. La concepción que presenta Bañuelos (2002) sobre la Resolución de Situaciones Matemáticas es la siguiente:

(...) el proceso de resolución de problemas supone la entrada como la percepción del problema, la salida como la respuesta posible, y entre estas dos, el conocimiento de tipo declarativo y procedimental. A partir de esta percepción, se estructura el espacio del problema, que es el mecanismo que establece el estado inicial, la meta, las restricciones que deben tenerse en cuenta y las estrategias que se pondrán en juego (p. 1). 
De esta manera, se puede decir que el proceso cognitivo va a depender de la forma de organizar el medio que lo rodea y adaptar las habilidades cognitivas al estilo de aprendizaje de los alumnos con retraso mental. Esto es importante en la Resolución de Situaciones Matemáticas, ya que los estudiantes adquieren, procesan, almacenan y recuperan la información obtenida, de una forma más adecuada. Bañuelos (2002) continúa indicando que la resolución de situaciones es un proceso cognitivo complejo, que involucra el conocimiento almacenado en la memoria a corto y largo plazo y la aplicación de este en distintos contextos.

Se considera que las estrategias de Resolución de Situaciones Matemáticas ayudan a interpretar las situaciones, a localizar el conocimiento y los procedimientos almacenados y a generar nuevas relaciones para llegar a la meta planteada, identificando las soluciones y corrigiendo los errores con la mediación docente.

Promover en los niños el aprendizaje de las matemáticas implica ayudarles a agilizar su memoria e inteligencia para generar nueva información haciendo uso de acciones mentales. Pero en los niños con problemas de aprendizaje, se observan dificultades que, como indican Dockrell y McShane (1997), hacen "que muestren un ritmo de aprendizaje más lento y alcancen un techo más bajo" (p. 162). Muchos de estos niños no logran el nivel esperado de conocimiento, ni emplean adecuadamente su memoria a corto o largo plazo, lo que implica dificultades para procesar y adquirir información y, por ende, poco conocimiento de su aprendizaje. Otro obstáculo que se refleja en esta población es la poca concentración o atención en su funcionamiento cognitivo, situación que provoca una incorrecta ejecución de situaciones matemáticas que no permiten una realimentación de lo aprendido.

Entre los principales teóricos de la Resolución de Situaciones Matemáticas que han aportado una serie de estrategias, se encuentran: Polya, Schoenfeld y Kantowsky, citados por Bañuelos (2002).

Para Polya, citado por Bañuelos (2002), un problema se resuelve correctamente si se siguen los siguientes pasos: comprender el problema, descubrir las relaciones entre los datos y la incógnita; si no se encuentra una relación inmediata, se deben tomar en cuenta situaciones auxiliares, así como tener un plan de resolución y cuando se haga, se podrá comprobar cada paso.

Los niños con retraso mental pueden comprender la situación planteada, ya sea leyendo o escuchándola, pero algunos no la podrán resolver, ya que se les dificulta comprender las preguntas y relacionar los datos, así que buscarán la manera de obtener un resultado satisfactorio con la información que ellos mismos poseen, tratando de dar una respuesta, aunque no sea la correcta. 
Cuanto más precisa sea la información para el niño, más fácil le resulta afrontar una situación. Solucionar una situación matemática involucra variables propias de la persona (motivación, habilidades matemáticas, sexo, estilo de aprendizaje), del entorno que se relaciona de alguna forma con la presentación de la situación (con información relevante o irrelevante, si es de forma gráfica, simbólica, textual o combinada) y de la representación de la situación que implica identificar y comprender lo que se pide.

La Resolución de Situaciones Matemáticas se refiere a la elaboración de estrategias utilizando recursos como conteo, cálculo mental, estimación, analogías, operaciones aritméticas, entre otros. Cuando el estudiante tenga una mejor comprensión de los conceptos, podrá ampliar sus estructuras tomando conciencia de los pasos y las estrategias que requiere para llegar a la solución de la situación matemática planteada.

\section{Retraso mental}

A través del tiempo, la concepción de retraso mental ha tenido diferentes interpretaciones, que responden a cambios en los paradigmas de la educación especial. El concepto de retraso mental aportado por Verdugo (1994) es el siguiente:

Retraso Mental hace referencia a limitaciones sustanciales en el funcionamiento actual. Se caracteriza por un funcionamiento intelectual significativamente inferior a la media, que generalmente coexiste junto a limitaciones en dos o más de las siguientes áreas de habilidades de adaptación: comunicación, autocuidado, autodirección, salud y seguridad, habilidades académicas funcionales, tiempo libre y trabajo. El retraso mental se ha de manifestar antes de los 18 años de edad (p. 20).

El concepto de retraso mental ha variado a través de los años, tratando de ajustarse a un concepto más exacto que incluya a cualquier persona con esta condición. En la actualidad, se habla de otros términos para referirse a esta población, tales como, "discapacidad cognitiva", "compromiso cognitivo"; sin embargo, para efectos del estudio, el grupo de investigación adopta la concepción dada por Verdugo. Asimismo, el retraso mental puede darse por múltiples causas tanto en los factores orgánicos o biológicos, como en los factores sociales (Barquero, González, Navarro y Vázquez, 1997).

Es importante considerar que este paradigma de retraso mental (Verdugo, 1994) plantea una clasificación basada en la intensidad de los apoyos que debe 
recibir esta población, según sean sus características de empleo en tiempo y duración. Dichos apoyos van desde los intermitentes, los limitados, los extensivos, hasta los generalizados y su utilización tiene relación con el grado de compromiso cognitivo, o bien, con las áreas de habilidades adaptativas que estén comprometidas.

De esta manera, es importante tomar en cuenta que cada persona con retraso mental necesitará o requerirá de diferentes apoyos debido a la diversidad presente en sus necesidades, lo que le ayudará a obtener resultados positivos en la vida cotidiana.

\section{Marco metodológico}

El tipo de diseño empleado en la investigación es cualitativo; se necesita que los niños expresen en forma oral cada uno de los pasos y procesos que utilizan en la Resolución de Situaciones Matemáticas.

Se resumen el registro y descripción detallados de sus expresiones, para luego, categorizar los procesos tanto individual como grupal. Krause, citado por Vizcarra (1999), indica que en la mayoría de los diseños cualitativos “...la selección de la muestra, la recolección de datos, el análisis y la generación de resultados están en una relación recíproca. En la práctica esta interacción implica la implementación simultánea de dichos procedimientos, incluyendo la generación paulatina de resultados" (pp. 13, 14).

Un estudio de caso es profundizar en la relación entre los participantes y las investigadoras (Salduondo, 2000). Este estudio se define como un conjunto de procedimientos de investigación necesarios de abordar en profundidad y buscar conclusiones que permitan comprender mejor el funcionamiento de los procesos cognitivos en personas con retraso mental. El tipo de observación utilizado fue no participante.

La unidad de análisis está conformada por dos escuelas que se seleccionaron a conveniencia. Estas son instituciones públicas del Ministerio de Educación, en las que funciona el servicio de Aula Integrada de retraso mental, las cuales fueron tomadas para el trabajo de campo. Una de las instituciones fue la Escuela Juan Mora Fernández, ubicada en Santa Bárbara de Heredia, circuito 03 y la otra fue la Escuela Líder Pacto del Jocote en Alajuela, circuito 04, donde laboran dos de las investigadoras. Ambas instituciones cuentan con más de mil estudiantes, en las que además de las materias básicas, se reciben otras como música, religión, inglés y artes plásticas. Tienen laboratorios de cómputo dados por la Fundación Omar Dengo, servicio de biblioteca, comedor y gimnasio. Por otra parte, en estas escuelas funcionan diversos servicios de educación especial, 
como por ejemplo, aula recurso, terapia de lenguaje y apoyo itinerante para diferentes discapacidades.

Los niños seleccionados para el estudio debían tener una adecuada expresión verbal y seguir las instrucciones. Las características que presentaban eran las siguientes: estudiantes con retraso mental, diez mujeres y diez varones con edades entre los 8 y 14 años, ubicados en los diferentes niveles de funcionamiento (inicial, intermedio y avanzado).

Los niveles de funcionamiento se distribuyeron de la siguiente forma: en el nivel inicial (I) seis niños (un niño y cinco niñas), en el nivel intermedio (M) seis niños (una niña y cinco niños) y en el nivel avanzado (A) ocho niños (tres niñas y cinco niños). El nivel socioeconómico es de medio bajo a bajo y la interacción entre estudiantes no se dio, ya que la aplicación del instrumento se realizó individualmente y la interacción alumno-docente manifestó confianza, tranquilidad y respeto.

Instrumentos de recolección y registros de datos: Para este fin, se diseñaron estrategias metodológicas que consisten en una serie de situaciones matemáticas ajustadas a la edad, nivel de funcionamiento académico y nivel socioeconómico de los veinte niños con los que se trabajó. Estas estrategias fueron construidas de forma que, cuando fueran aplicadas, reflejaran los procesos cognitivos en los niños con retraso mental. Su estructura cumple con distintas partes: una ficha ilustrada, una ficha de trabajo y una guía de observación. También se elaboró un instructivo con toda la información necesaria, para que las personas que las aplicaran no tuvieran dificultad.

Cada una de las estrategias está elaborada para que, mediante imágenes y preguntas, el niño tome conciencia de lo que realiza y las formas que emplea para resolver la situación matemática planteada (suma o resta). Se empleó este tipo de estrategia como instrumento, pues lo que se buscaba era enfrentar al niño a una situación de aprendizaje, en la que pudiera exteriorizar cada uno de los pasos que seguía para solucionar la situación matemática que se le presentaba y que evidenciara así los procesos empleados.

Primero, se elaboró una serie de situaciones matemáticas que sirvieron para determinar los conocimientos previos que manejaban los niños respecto a la Resolución de Situaciones Matemáticas, con el fin de saber el manejo real que tenían de los contenidos matemáticos. Este primer instrumento fue sometido a criterios de jueces y a pruebas pilotos. La información recopilada sirvió para construir la Batería de Resolución de Situaciones Matemáticas para Niños con Retardo Mental (BARSIMAR). Este instrumento fue diseñado para obtener la información, empleando estrategias de metacognición y ajustándolo a 
las características de cada uno de los niños y sus niveles de funcionamiento: inicial (I), intermedio (M) y avanzado (A). Este instrumento fue importante para detectar los procesos metacognitivos y observar como se desenvuelven los niños al desarrollar las situaciones matemáticas.

Validación de la Batería: Para la validación de la Batería, se efectuaron varias pruebas pilotos con otros niños de otras Aulas Integradas, que no fueran los participantes del estudio. Se les aplicó la BARSIMAR de forma individual y en las mismas condiciones que requiere el instrumento. Del resultado de las sugerencias, aportes y ajustes realizados, se construyó la versión final. También se sometió a criterios de jueces y se consideró la opinión de especialistas tanto en Evaluación como en Matemáticas, Metacognición y docentes de Aula Integrada.

La BARSIMAR fue sometida a criterios de rigor desde una perspectiva cualitativa para su validación. Por lo tanto, la validación de este instrumento es únicamente para efectos del estudio y no puede ser generalizada. Por esta razón, se recomienda ajustar el instrumento a las necesidades y características de los estudiantes a los que se aplicará.

Es importante destacar que la BARSIMAR está dirigida a niños con retraso mental, que no posean otro tipo de discapacidad asociada como podrían ser las deficiencias visuales, ya que el tipo y tamaño de letra no están adaptados a dichas necesidades. La BARSIMAR presenta apoyo de material de lo concreto a lo abstracto, apoyo visual y gráfico en colores y espacios de trabajo amplios para facilitar el desarrollo y ejecución de cada una de las estrategias matemáticas.

Las estrategias metacognitivas en las que se basa la BARSIMAR se crearon de acuerdo con el proceso de enseñanza y aprendizaje, comprendido como una estructuración, que va de lo más simple a lo más complejo, mediante actividades en las que el niño logre interiorizar los conceptos aprendidos (Marín, 2000).

La persona que aplica la BARSIMAR se constituirá en un mediador que guía cada paso, tomando en cuenta que, conforme los niños avanzan de nivel, requerirán mayor independencia en la ejecución y resolución de cada situación matemática planteada.

El propósito de la BARSIMAR es precisamente hacer que los niños puedan expresar abierta y claramente los pasos y procesos cognitivos que necesitan para solucionar el problema que se les plantea. Los contenidos de dichos problemas, además de basarse en los conocimientos previos de los participantes, tienen mucha relación con contenidos de aplicación práctica y de la vida cotidiana. En cada sesión, se les solicitó a los niños que explicaran cómo iban a 
resolver la situación, qué pasos siguieron, qué necesitaban saber para resolverla adecuadamente y cómo enfrentaban la situación de aprendizaje ${ }^{5}$.

Los datos de la sesión de trabajo con cada niño fueron grabados en una cinta de audio y posteriormente transcritos, después se extrajeron los "codes" (palabras que se obtienen como resultado de la codificación del texto) para construir las categorías del análisis posterior.

Instrucciones: La administración de la BARSIMAR se realizó de manera individual (al lado o de frente al niño), cada aplicador estableció un espacio físico apropiado, libre de distractores y con los materiales necesarios: una ficha ilustrada con situaciones matemáticas (la que se muestra en todo momento al niño), una ficha de trabajo, un lápiz, un borrador, un tajador, material concreto (fichas, chapas, paletas, ábaco, frijoles, entre otros). Luego el aplicador o el niño leyó las preguntas una por una y este las resolvió en su ficha de trabajo. Conforme se desarrolló cada situación, se le hicieron diferentes preguntas que lo indujeron a expresar las acciones que ejecutó o llevó a cabo.

Los niños observaron la Ficha Ilustrada de Situaciones Matemáticas, según el nivel que les correspondía, luego se ejecutó el proceso en cada ficha de trabajo. Quien aplicaba la BARSIMAR podía anotar sus observaciones al final del documento (para que ellos no se inhibieran) en una ficha determinada para ese fin ${ }^{6}$. El observador anotó en los espacios correspondientes toda la información que logró apreciar en el niño referente a la ejecución de los procesos cognitivos. Esta información, junto con la obtenida en las transcripciones, sirvieron para la respectiva valoración de la BARSIMAR.

Evaluación de la Batería: En la BARSIMAR no se estableció ningún puntaje, ya que el interés era conocer y describir los procesos cognitivos que emplearon los niños para resolver cada situación matemática, por esta razón la interpretación de la información se hizo cualitativamente.

Según los niveles de funcionamiento (inicial, intermedio o avanzado) donde se ubica el niño, se realizaba una serie de preguntas para determinar los procesos cognitivos durante la aplicación de la BARSIMAR.

\footnotetext{
${ }^{5}$ Es importante destacar que, para la aplicación de las estrategias metodológicas propuestas, se solicitó permiso tanto a los directores correspondientes de cada institución seleccionada, como a cada uno de los padres de familia de los niños con los que se trabajó. Se efectuó una reunión de padres en la que se les comunicó el propósito del estudio, la importancia de la participación en este, el tiempo que tomaría el estudio, el respeto por el anonimato y la confiabilidad en el manejo de la información, así como el compromiso en la devolución de los datos.

${ }^{6}$ Lo ideal es que la hoja de observación sea llenada por otra persona ajena a la situación (observador no participante), que no sea el aplicador de la BARSIMAR, con el fin de obtener una mejor información tanto de las actitudes como de las formas de expresión verbal de los niños.
} 
Es importante reafirmar y estimular en todo momento los logros obtenidos por medio de frases positivas, gestos, palmadas, entre otros incentivos, que animen al niño a continuar resolviendo las situaciones matemáticas planteadas.

Una forma de valorar la información obtenida fue grabando cada sesión de trabajo con el niño, para posteriormente transcribirla. Esos datos, junto con las fichas de observación y las fichas de trabajo, se emplearon para obtener de manera más objetiva tanto los procesos cognitivos que usaron los niños al resolver las situaciones matemáticas, como las formas de expresión oral y las actitudes demostradas durante la aplicación de la BARSIMAR. Finalmente se resumió toda la información y se obtuvieron datos valiosos que ayudaron a comprender mejor a los estudiantes, así como ajustar estrategias matemáticas adecuadas y mejorar, de esta manera, su práctica educativa.

\section{Detección de los procesos cognitivos}

La categoría principal del análisis la constituyen los procesos cognitivos empleados por los niños en la resolución de las situaciones planteadas. Se detectaron otras categorías de análisis: conocimientos matemáticos, ejecución de la tarea, dificultades que presentaron los niños en la aplicación de cada una de las situaciones matemáticas, aspectos socioemocionales, actitudes demostradas y formas de expresión.

A continuación, se presentan los resultados generales obtenidos en la investigación, ordenados según los niveles de funcionamiento y por categorías de análisis.

Los sujetos de estudio del nivel inicial no logran almacenar la información por largo tiempo. Por lo general, esta es retenida en la memoria a corto plazo y luego decae y se produce el olvido. Esto hace que la memoria a largo plazo prácticamente no se active, lo que lleva al niño a recuperar mal la información que dispone o a organizar respuestas incorrectas producto de interferencias. Todo ello lleva a malas ejecuciones, por lo tanto, a la ausencia de una realimentación adecuada. En el nivel intermedio, la mayoría de los niños no lograron almacenar la información en la memoria a corto plazo, sino más bien tienden a realizar una búsqueda incorrecta y de forma automática en la memoria a largo plazo, lo que provoca que a la hora de reconstruir el conocimiento se presente mucha interferencia, en consecuencia, la respuesta no es organizada adecuadamente. En el nivel avanzado, la información logró ser almacenada en la memoria a corto plazo; sin embargo, al buscar y recuperar la información en dicha memoria, no pueden organizarla correctamente, ya que se presentan interferencias que hacen que la información se decaiga y con facilidad llegue al 
olvido. Se maneja la información en la memoria a largo plazo, pero no logra ser recuperada ni organizada en forma adecuada, ya que no se sienten seguros de confirmar y verificar lo aprendido. En estos niños se observan mejor los procesos cognitivos, pero no logran ejecutarlos de forma continua y adecuada.

\section{Análisis general de los procesos cognitivos}

$\mathrm{Al}$ analizar los tres niveles, se observó que los estudiantes principalmente en los niveles inicial e intermedio muestran dificultades en los procesos cognitivos, porque presentan mucha interferencia o decaimiento, desde que perciben y reciben la información, la cual no ha sido bien procesada o grabada en la memoria a largo plazo. Esta situación se da debido a la falta de atención, concentración, disposición, motivación, inseguridad, entre otras características.

Al tener problemas de codificación semántica, la memoria a largo plazo está afectada. También, al no comprender los significados de lo que está leyendo, oyendo o viendo, no puede guardar la información correctamente. En el nivel avanzado, los procesos cognitivos, como la recepción y percepción de los estímulos y codificación semántica, no presentan dificultades para procesar la información en la memoria a largo plazo, ya que los niños demostraron actitudes positivas ante las situaciones presentadas y tenían conocimientos sobre lo que estaban realizando (significado).

En los tres niveles, se observó que los niños, en la memoria a largo plazo, tienden a verbalizar lo que están realizando, para ejecutar la situación. Además de realizar la búsqueda y recuperación de forma automática, con lo que provocan una reconstrucción de conocimientos con mucha interferencia, por lo que la respuesta no es organizada adecuadamente.

En el nivel inicial, los niños no logran llegar al proceso de realimentación y verificación, debido a la organización incorrecta de respuesta que obtuvieron. Asimismo, en los niveles intermedio y avanzado no todos los niños lograron llegar al proceso de realimentación y verificación, posiblemente, por la dificultad para organizar la respuesta en forma correcta. Se puede decir, que las dificultades como aspectos socioemocionales, actitudes y formas de expresión oral que demostraron los estudiantes, afectaron los conocimientos matemáticos y la ejecución de la tarea.

Al respecto, Kirk y Gallagher (1989) (citados por García et al., 1996) consideran que los niños presentan dificultades, en especial, en las áreas de percepción, operaciones mentales, expresión, toma de decisiones. Se pudo detectar que las mismas dificultades presentaron los participantes del estudio; al estar estas áreas afectadas, los procesos cognitivos tienen mayor dificultad y no logran la ejecución de situaciones matemáticas. 
No hay una adecuada regulación y autocontrol de las tareas de aprendizaje de estos niños, debido a que no planifican lo que van a realizar; además, pueden ejecutar intentos de monitoreo en la tarea y expresar: "déjeme pensar", "espere para ver", "esto está mal", entre otras frases.

Según Moya (2000a), la atención depende de lo que la persona quiera atender y de los estados de conciencia, mejora con la práctica y el entrenamiento. Esta situación se observó cuando los niños tenían problemas para identificar los elementos que estaban en la ficha de trabajo de la BARSIMAR; entre más dificultad encontraban para seleccionar los datos iniciales, mayor dificultad tenían para resolver adecuadamente la situación matemática.

Esto hace pensar en la importancia que tienen el tipo de material y el estímulo que se le presenten a los niños con retraso mental para resolver situaciones matemáticas, ya que si se logra captar la atención del estudiante, mejor va a recibir la información y, por lo tanto, procesarla en forma correcta.

Por otra parte, cuanto mayor sea el entrenamiento y la exposición de estrategias metacognitivas, mejor capacidad tendrán los niños para tomar conciencia de los procesos que necesitan para procesar la información.

\section{Resultados}

Se comprobó la eficacia de las estrategias de metacognición en la Resolución de Situaciones Matemáticas en los niños con retraso mental, ya que se les dio la oportunidad de tener una experiencia donde utilizaron sus propios conocimientos.

Se logró detectar en los conocimientos previos, que en el nivel inicial los niños realizan lectura, operatoria e identifican los números del uno al diez; en el nivel intermedio ejecutan operatoria de suma, lectura e identifican cantidades del uno al cincuenta y en el nivel avanzado los estudiantes hicieron la operatoria, lectura de cantidades de tres y cuatro dígitos.

En la aplicación del instrumento, se observó que los conocimientos previos en los tres niveles de funcionamiento son diferentes, ya que es una población que presenta características heterogéneas como: edad, conocimientos, habilidades, discapacidad, entre otras.

Los procesos cognitivos que los niños emplean en los tres niveles de funcionamiento no son los mismos, pues el procesamiento de la información es realizado de diferente manera. Por ejemplo, los niños del nivel inicial tienen dificultad al recibir la información, al desconocer algunos términos, no leen, no conocen números, cantidades ni sumatoria; esta información se queda en la memoria a corto plazo, situación que no permite obtener respuestas correctas o 
explicar el proceso que realizó. Los estudiantes de los niveles intermedio y avanzado, que resuelven las situaciones matemáticas, lo hacen mecánicamente o razonando; algunos llegan al almacenamiento en la memoria a largo plazo y otros a la realimentación, razón que permite dar respuestas correctas, aunque con interferencias en la mayoría de los casos.

En las habilidades de metacognición, los niños presentaron dificultades para desarrollarlas, posiblemente debido a su discapacidad y a la forma en que se les ha enseñado a resolver situaciones matemáticas. Para lograr que esta población resuelva este tipo de situaciones, es necesario enseñarles a desarrollar la capacidad para adquirir la información, que se logra utilizando estrategias y procedimientos que estimulen su aprendizaje.

Los pasos que emplearon al resolver situaciones matemáticas fueron: observar el material, colocar la operación y el signo, realizar la operación y dar la respuesta a la tarea solicitada. Algunos niños solicitaban ayuda cuando se les dificultaba resolver las situaciones matemáticas, ya que no se sentían seguros de lo que estaban haciendo, esto por no leer y comprender los enunciados y no retener la información, situación que se presenta en la mayoría de los casos, especialmente en el nivel inicial.

Los estudiantes manifestaron interés, curiosidad, gusto y motivación por las estrategias metacognitivas en la Resolución de Situaciones Matemáticas. Así lo expresaron cuando se les preguntó si les gustaría trabajar de esa manera. Esa actitud debe aprovecharse para realizar constantemente ejercicios que los motiven y poco a poco, esa información pase a formar parte de la que ya poseen, lo cual genera nuevos conocimientos que pueden aplicar en su vida cotidiana.

El tiempo que emplearon los niños para resolver las situaciones matemáticas fue de 5 a 15 minutos, dependiendo del caso, lo cual debe considerarse a la hora de plantear las situaciones para que el tiempo sea bien aprovechado y retengan la mayor cantidad de información posible. La mayor dificultad que presentaron al resolver las situaciones matemáticas fue la operatoria de la resta, como colocar la operación y el signo, realizar la operación y llegar a la respuesta, debido a que no tenían claro su concepto. Esta operación debe trabajarse más con ellos.

Los docentes deben prepararse para aplicar estrategias metacognitivas, que estimulen el aprendizaje de los niños en las diferentes situaciones matemáticas y en las demás áreas. Estas estrategias de Resolución de Situaciones Matemáticas permiten que los niños pongan en práctica su creatividad, expresividad e imaginación en la tarea cognitiva.

Las estrategias metacognitivas en la Resolución de Situaciones Matemáticas son de gran importancia, ya que los niños comprenden el significado del aprendizaje de las matemáticas y les permiten resolver situaciones cotidianas. 
El estímulo de estrategias metacognitivas brinda la oportunidad de desarrollar el pensamiento cognitivo de los estudiantes. Esta situación se observó en los resultados obtenidos de la BARSIMAR.

Estos procesos cognitivos muestran diferencias entre los tres niveles (I, M, A); se presenta una mayor dificultad en el nivel inicial, donde logran llegar al almacenamiento de la información en la memoria a corto plazo, la que se ve afectada por interferencia y decaimiento; situación que los lleva rápidamente al olvido.

En el caso de los otros niveles de funcionamiento, logran llegar al almacenamiento en la memoria a largo plazo y en algunos casos a la retroalimentación y refuerzo. Este resultado hace ver que estos niños pueden aprender a resolver diferentes situaciones si se les enseña como hacerlo, de aquí la propuesta de utilizar diferentes estrategias metacognitivas para conseguir buenos resultados en la enseñanza de su aprendizaje.

\section{Recomendaciones}

Para el Ministerio de Educación Pública (MEP): - Fomentar estrategias metacognitivas en el programa educativo, en la atención de los niños con necesidades educativas especiales, para un mejor aprendizaje. - Brindar capacitaciones a los docentes sobre la metacognición. - Promover el intercambio de información en las instituciones educativas sobre la metacognición, mediante conferencias o seminarios relacionados con el uso de estrategias metacognitivas en la Resolución de Situaciones Matemáticas o en otras áreas, en niños con retraso mental.

Para el docente: - Es necesario que se preparen en el campo de la metacognición en la Resolución de Situaciones Matemáticas, con el fin de propiciar en los estudiantes con retraso mental un aprendizaje más significativo. - Se debe permitir que el estudiante pregunte y experimente haciendo uso del material propuesto. - El material que se ofrece a los alumnos con retraso mental debe ser ilustrado, llamativo, para mediar y orientarlos a la Resolución de Situaciones Matemáticas. - Los docentes deben tomar en cuenta los pasos que el estudiante realiza cuando soluciona una situación matemática y no en el producto o el resultado, ya que muchas veces se centran solo en que la respuesta sea correcta y no se guía al niño para que aprenda a resolver las situaciones para desenvolverse independientemente. - Es importante que el docente les brinde a los alumnos estrategias metacognitivas adecuadas, para que ellos desarrollen el pensamiento y haya un proceso de aprendizaje más real y significativo. —Se debe 
tomar conciencia del propio proceso de enseñanza en la aplicación de las estrategias metacognitivas para resolver las diferentes situaciones, ya sean de la vida cotidiana o matemáticas, que se le presenten a sus estudiantes, para que el aprendizaje sea más provechoso y eficaz.

Para la Universidad Nacional, Educación General Básica (EGB): —Considerando los resultados obtenidos en el uso de metacognición en la Resolución de Situaciones Matemáticas, es importante realizar investigaciones que permitan crear estrategias metacognitivas, para niños con retraso mental y determinar el tipo de metodología que emplean los docentes en la enseñanza de este tipo de situaciones. - A los estudiantes que se están preparando en Educación se les debería formar en Metacognición, para que preparen a los niños a aprender en la Resolución de Situaciones Matemáticas y en las diferentes áreas de estudio. - En los cursos de matemática que se brindan a los docentes en formación, es importante que estos tengan claro, ¿cómo aprende el niño? y no ¿qué debe aprender? -Es importante que en el curso Didáctica de la Matemática se realicen instrumentos con estrategias metacognitivas para la Resolución de Situaciones Matemáticas, pues permiten ampliar sus conocimientos. 


\section{Referencias}

Acle, G. y Olmos, A. (1998). Problemas de Aprendizaje. Enfoques Teóricos. Segunda edición. México D.F.: Universidad Autónoma de México.

Bañuelos, A. (2002). Resolución de Problemas Matemáticos en Estudiantes de Bachillerato. En línea http://www.cesu.unam.mx/iresie/revistàs/perfiles/perfiles-ant/ 67-6.htm

Barquero, D., González, E., Navarro, F. y Vázquez, P. (1997). El Programa de Comunicación Total de Schaeffer y Colaboradores (1982): "Habla signada" como un sistema alternativo en la enseñanza del lenguaje en niños con retraso mental no verbales. Seminario de Graduación, Universidad Nacional, Heredia.

Cordero, M., Duarte, L., Fuentes, O., López, N. y Vidal, M. (1997). La Resolución de Problemas para el Aprendizaje de las Matemáticas. Seminario, Universidad Nacional, Heredia, Costa Rica.

Dockrell, J. y McShane, J. (1997). Dificultades de Aprendizaje en la Infancia. Barcelona, España: Ediciones Paidós Ibérica.

Escudero, C. (2002). Los Procedimientos en Resolución de Problemas de Alumnos de $3^{\circ}$ Año: Caracterización a través de Entrevistas. San Juan, Argentina. En línea http://www.if.ufrgs.br/public/ensino/N3/proced.htm

García, M., Montero, S., Romero, C. y Salvador, L. (1996). Evaluación Psicosocial del Retraso Mental, Conceptos, Metodología e Instrumentos. Grupo de Investigación en Medicina Psicosocial. Universidad de Cádiz. Madrid, España: Ediciones Ministerio de Asuntos Sociales.

González, F. (s.f.). Metacognición y Tareas Intelectuales Existentes. El Caso de la Resolución de Problemas Matemáticos. En línea: http://www.lite.fae.unicamp.br/ grupos/matema/zete/Z9 fredy.html

Klinger, K. (1999). Psicología Cognitiva: Estrategias en la Práctica Docente. España: Espasa-Calpe.

Moya, J. (2000a). Estrategias Metacognitivas de Educación Especial. Pontificia Universidad Católica de Chile. Santiago: PUC.

Moya, J. (2000b). Estrategias Metacognitivas y Dificultades del Aprendizaje. Pontificia Universidad Católica de Chile. Santiago: PUC.

Salduondo, J. (2000). Entre lo Visible y lo Enunciable. El Libro de Texto en la Escuela Básica Chilena. Algunos Lineamientos Estratégicos para Incidir en el Mejoramiento de las Prácticas Educativas. Seminario de Investigación para obtener el grado de Magíster. Pontificia Universidad Católica de Chile.

Thomton, S. (2000). La Resolución Infantil de Problemas. Segunda edición. Madrid, España: Ediciones Morata.

Verdugo, M. (1994). Personas con Discapacidad, Perspectivas Psicológicas y Rehabilitadoras. Madrid, España: Editorial Siglo XXI.

Vizcarra, R. (1999). Teoría Fundada: Codificación Abierta y Análisis Comparativo. Pontificia Universidad Católica de Chile, Facultad de Educación. 